\title{
Identification of Novel Small Molecules That Bind to the Loop2 Region of Sclerostin - an in silico Computational Analysis
}

\author{
K. MUTHUSAMY ${ }^{1}$, S. MOHAN ${ }^{2}$, S. NAGAMANI ${ }^{1}$, C. KESAVAN ${ }^{2}$ \\ ${ }^{1}$ Department of Bioinformatics, Alagappa University, Karaikudi, India, ${ }^{2}$ Musculoskeletal Disease \\ Center, VA Loma Linda Healthcare system, Loma Linda, CA, USA
}

Received November 24, 2015

Accepted February 18, 2016

On-line July 15, 2016

\section{Summary}

The goal of this study was to identify small molecular weight compounds that bind to sclerostin using in silico methods because of the established importance of sclerostin-based therapies for the treatment of disease characterized by low bone mass. The zinc database $(\mathrm{Zdb})$ revealed that nine potential molecules bind to the loop2 region (functional site) of sclerostin with $A D M E / T$ properties that are within an acceptable range defined for human use. Compounds 30160056 and 56871042 showed the highest docking score. Density functional theory (by HOMO, LUMO and MESP analysis) and MM/GBSA analysis showed that four compounds 30160056, 56871042, 72112226 and 43920281 exhibit high stability among the nine small molecules identified. Induced Docking Fit and Pymol software analyses revealed that the identified compounds differ in the interaction with amino acids in the loop2 region of sclerostin. Six compound exhibited interaction with Ile95 and 2 compounds with Asn93, an amino acid in the loop2 region known to be involved in sclerostin's inhibitory effect, suggesting that the identified compounds have the potential to bind and neutralize sclerostin function. Furthermore, compound 43920281 showed a low risk of toxicity and drug-like characteristic features compared to all nine identified compounds. In conclusion, in silico analysis identified a novel compound 43920281 as a potent anti-sclerostin therapeutic for drug development for the treatment of osteoporosis.

\section{Key words}

Osteoporosis • Sclerostin (SOST) • Wnt (wingless-type MMTV integration site family) antagonist • Computer-aided drug design (CADD)

\section{Corresponding author}

C. Kesavan, Musculoskeletal Disease Center, VA Loma Linda Healthcare System, Research Service, 11201 Benton St, Loma Linda, CA 92357, USA. Fax: 011909796 1680. E-mail: Chandrasekhar.Kesavan@va.gov

Osteoporosis is a disease characterized by low bone mineral density (BMD) and poor bone quality resulting in reduced bone strength and an increased risk of bone fractures (Drake et al. 2015, Kobayashi and Kronenberg 2014, Lombardi et al. 2010, Olney 2009). To date, millions of individuals suffer from osteoporosis due to a variety of causes including aging, lack of exercise and endocrine/ metabolic disturbances (Golob and Laya 2015, Preisinger et al. 1996). According to the "National Osteoporosis Foundation", osteoporosis is responsible for two million broken bones and $\$ 19$ million in related healthcare costs every year, and by 2025 the bone fracture numbers are expected to raise and the annual associated costs to increase to 25.5 billion. Currently, moderate exercise, vitamin based therapy and parathyroid hormone (PTH), a Food and Drug administration (FDA) established bone anabolic therapeutic agent, that helps to maintain bone mass and reduce the incidence of osteoporosis (Augustine and Horwitz 2013, Bhutani and Gupta 2013, Iwamoto 2014, Laktasic-Zerjavic 2014, Prentice 2004), however the amount of new bone formation stimulated with these agents is less effective in individuals who have lost a tremendous amount of bone (postmenopausal women and in the elderly). Therefore, there is a need for the identification and development of 
additional agents as therapeutics to stimulate new bone formation not only to reduce the incidence of osteoporosis but also to treat bone injuries.

In osteoporosis, higher osteoclast activity enhances bone resorption and reduces osteoblast activity, which reduces bone formation, are major causes for the pathogenesis of osteoporosis. Recent studies have shown that sclerostin, a protein encoded by the SOST gene, expressed in osteocytes, is a key player in the pathophysiology of osteoporosis. Studies using animal and human models have delineated that mutations in the sclerostin gene resulted in increased bone mass and bone strength (de Vernejoul and Kornak 2010, Li et al. 2008). Subsequently, clinical studies revealed that postmenopausal women with decreased bone mass and increased bone loss showed high levels of sclerostin in the serum (Ardawi et al. 2012). In terms of underlying mechanisms to account for bone loss, studies using in vitro and in vivo models have shown that Sclerostin binds to Low density lipoprotein (LRP) 5/6 and thereby inhibits wingless (WNT) signaling, a pathway well established in promoting new bone formation (Kim et al. 2013). Likewise, other studies have shown that Sclerostin blocks the osteogenic effect induced by blocking Bone Morphogenic Protein (BMP) ligand interaction with BMP receptors (van Bezooijen et al. 2007, Winkler et al. 2003). These data from independent studies led to the conclusion that sclerostin is a key contributor to osteoporosis and that blocking sclerostin should increase new bone formation. Accordingly, animal and human model studies have provided evidence that blocking sclerostin using antibody based therapies promoted new bone formation and reduced the risk of osteoporosis (Clarke 2014, Lewiecki 2011). Although antibody based therapies have been efficient, this type of therapy is expensive and thus has a significant impact on health care costs. Therefore identifying novel therapeutics that are less costly and show a potential for blocking sclerostin function will have a significant impact not only in reducing the detrimental effects of osteoporosis on our aging population but also on future health care costs. Therefore, we undertook a computational analysis using in silico and molecular docking analyses to identify small molecules that could inhibit sclerostin actions.

All computational analyses were carried out on a Red hat 5.1 Linux platform running on a Lenovo Intel core 2 duo processor with 2 GB of RAM. We used different chemical databases such as the Asinex (www.asinex.com) (55958 compounds), Chembridge (www.chembridge.com) (511324 compounds) and Zinc databases (www.zinc.docking.org) (155819 compounds) to identify molecules that dock into the active site of sclerostin to screen compounds which could inhibit sclerostin actions. The crystal structure of sclerostin (Protein database (PDB) id - 2k8p) was downloaded from the PDB (Veverka et al. 2009) and prepared using the 'protein preparation module' (Schrödinger, LLC, New York, 2011). In order to evaluate the overlapping atoms, Hydrogen atoms were added to the crystal structure. Furthermore, all the "structural" waters molecules were removed since water molecules have not been shown to be critical to the function of the proteinligand interaction. Partial atomic charges were created due to the asymmetric distribution of electrons in chemical bonds. In order to determine the atomic charges, the Schrodinger software used the OPLS_2005 (optimized potentials liquid for simulations) force field, which contains different parameters for treating different atoms. Furthermore, the energy was minimized until the average root mean square deviation of non-hydrogen atoms reached $0.30 \AA$, to avoid structure deviations. Once the structure was prepared, a receptor grid was generated. The grid enclosing box was centered over the active amino acids (Proline (Pro)92, Asparagine (Asp)93, Alanine (Ala)94, Isoleucine (Ile)95 and glycine (Gly)96), represented as PNAIG, in the loop-2 region of sclerostin (Veverka et al. 2009) so that they were enclosed within $3 \AA$ from the center of the residues. The scaling Van der walls radii were set at $1.0 \AA$ in the receptor grid generation. The grid was enclosed with the bounding box set at $20 \AA$. A virtual screening workflow was used to screen the large collections of compounds against the defined target in the sclerostin molecule. This workflow included Ligprep (for ligand preparation, version 2.5, Schrodinger, LLC, New York, 2011), Qikprop (ADME/T (Adsorption Distribution Metabolism Excretion / Toxicity predictions, version 3.5, Schrodinger, LLC, New York, 2011), HTVS (high throughput virtual screening) and other structural properties (HTVS, Schrodinger, LLC, New York, 2011). Asinex, TOSLab collections and Zinc databases which contain a total of 723,101 compounds were screened based on Glide score, Glide energy and hydrogen bond interactions (Schrodinger). To further confirm the docking results, we re-ran the whole docking process using "Induced Fit Docking" (mixed molecular docking/molecular dynamics protocol) which keeps receptors flexible (Singh et al. 2012). In this protocol both the protein and ligand molecules were kept flexible. 
We set the same default parameters as mentioned in the normal Glide XP docking method. Subsequently, the prime MM/GBSA (Molecular Mechanics Generalized Born Surface Area) method was used to calculate the free energy of binding for a set of ligands to a receptor. The energy minimization was carried out using the local optimization feature in Prime, and the free energies of the complex were calculated using the OPLS_2005 force field program and GB/SA continuum solvent model. The binding free energy was calculated as follows (Das et al. 2009, Lyne et al. 2006),

$$
\begin{aligned}
& \Delta \mathrm{G}_{\text {bind }}=\Delta_{\mathrm{E}}+\Delta \mathrm{G}_{\text {solv }}+\Delta \mathrm{G}_{\mathrm{SA}} \\
& \Delta \mathrm{E}=\mathrm{E}_{\text {complex }}-\mathrm{E}_{\text {protein }}-\mathrm{E}_{\text {ligand }}
\end{aligned}
$$$$
\text { where } E_{\text {complex }}, E_{\text {protein }} \text { and } E_{\text {ligand }} \text { are the }
$$
minimized energies of the protein-ligand complex, protein and ligand, respectively.

$$
\begin{aligned}
& \Delta \mathrm{G}_{\text {solv }}=\mathrm{G}_{\text {solv(complex) }}-\mathrm{G}_{\text {solv(protein) }}-\mathrm{G}_{\text {solv(ligand) }} \\
& \text { where } \mathrm{G}_{\text {solv(complex) }}, \mathrm{G}_{\text {solv(protein) }} \text { and } \mathrm{G}_{\text {solv(ligand) }} \text { are }
\end{aligned}
$$
the solvation free energies of the complex, protein and ligand respectively.

$$
\begin{aligned}
& \Delta \mathrm{G}_{\mathrm{SA}}=\mathrm{G}_{\mathrm{SA} \text { (complex) }}-\mathrm{G}_{\mathrm{SA} \text { (protein) }}-\mathrm{G}_{\mathrm{SA} \text { (ligand) }} \\
& \text { where } \mathrm{G}_{\mathrm{SA} \text { (complex), }} \mathrm{G}_{\mathrm{SA} \text { (protein) }} \text { and } \mathrm{G}_{\mathrm{SA} \text { (ligand) }} \text { are the }
\end{aligned}
$$
surface area energies for the complex, protein and ligand respectively.

Additionally, all the identified compounds were subjected to density functional theory (DFT) calculations (Lee et al. 1988). DFT calculations are typically used for studying electronic molecular features to define a molecular electrostatic map, with the electron density, and frontier molecular orbital density fields (i.e. HOMO, LUMO) which can predict the molecular properties and biological activity of a compound. The DFT calculations were performed with Jaguar version 8.1 software, which sets the solvation state. The DFT was analyzed through Becke's three-parameter exchange potential and the Lee-Yang-Parr correlation functional (B3LYP) basic set at a 3-21G* level (Seminario 1996), using "Poisson Boltzmann Finite" (PBF) solvation. In the present study, the 3D-molecular electrostatic potentials (MESP) V(r) at a point $r$ because of a molecular system with nuclear charges located at and the electron density $\rho$ (r) were derived using the following equation,

$$
V(r)=\sum_{A=1}^{N} \frac{Z_{A}}{\left|r-R_{A}\right|}-\int \frac{\rho\left(r^{\prime}\right) d^{3} r^{\prime}}{\left|r-r^{\prime}\right|}
$$

In this equation $\mathrm{N}$ represents the total number of nuclei in the molecule and the two terms refer to the bare nuclear potential and the electronic contributions, respectively. We computed the Jaguar Dipole moment,
Molecular electrostatic properties, Lowest Unoccupied Molecular Orbital (LUMO), including MESP (Molecular electrostatic potential) and Highest Occupied Molecular Orbital (HOMO) energy. We calculated the electrostatic potentials by van der Waals contact surface area of the molecule (Jaquar, version 8.1, Schrodinger, LLC, New York, 2011).

To identify novel molecules that showed a high binding affinity to sclerostin, we used the published crystal structure of the human sclerostin protein (PBD id-2k8p) for the molecular docking analysis. In particular, we used the loop2 region of sclerostin because LRP5/6 binds to amino acids in the loop2 region of sclerostin, known as the PNAIG motif, thus, inhibiting LRP5/6 activity. LRP5/6 activity is required for activation of WNT signaling and subsequent bone formation. Additionally, a report has shown that a peptide derived from the loop2 region of sclerostin blocked the interaction of sclerostin with LRP5/6 (Holdsworth et al. 2012). Based on this information, we used the loop2 region of sclerostin to form a glide-grid for the screening analysis. Different databases including the Asinex, TOSLab and Zinc database were screened against the loop2 region of sclerostin using a virtual screening workflow in the maestro (Schrodinger, LLC, New York, 2009) for hit identification and lead compound optimization. The database ligands were prepared at $\mathrm{pH} 7.0 \pm 2.0$ using an epik state (enumerates ligand protonation states and tautomers in biological conditions) and the large penalties for high energy ionization of tautomer states were removed. In the default constraint parameters the protein has been kept as a scaling van der Walls radius of $1.0 \AA$ with a partial atomic charge set at less than $0.25 \AA$. The Glide HTVS was carried out with a flexible docking algorithm using selected constraints for each grid in the OPLS_2005 force field. We finalized the best lead molecules based on glide score rather than on glide energy. This is because glide energy includes only modified Coulomb-van der Waals interaction energy while the glide score includes other parameters such as hydrogen bond interactions, a lipophilic term and coulomb energy. The docking analysis revealed nine compounds that show a potential binding affinity towards the loop2 region of sclerostin (Table 1A). The 2D structure of each identified lead molecules is displayed in Figure 1 (supplement data). We further confirmed the docking analysis using an Induced Fit Docking analyses which revealed similar types of interactions. 

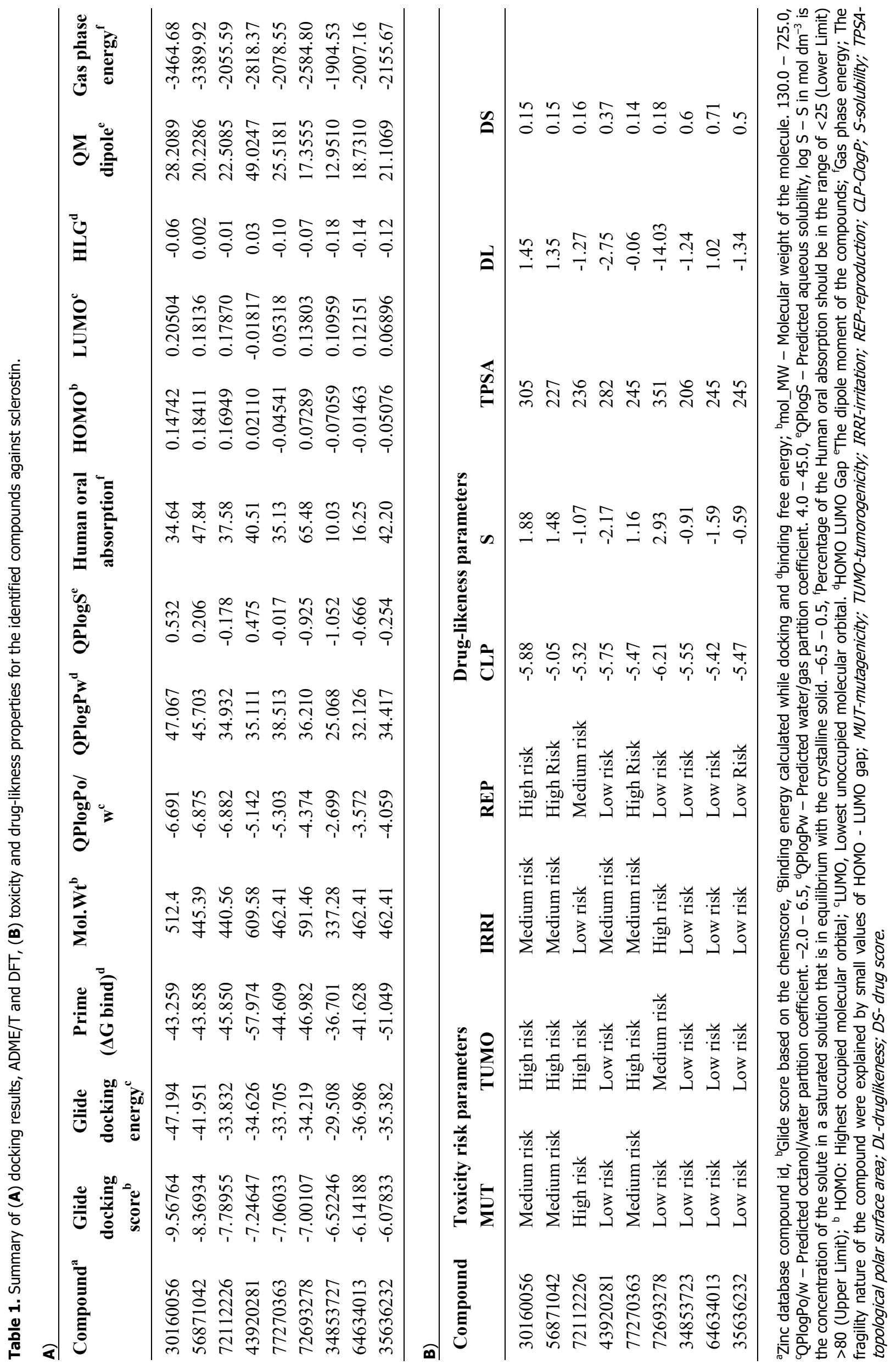


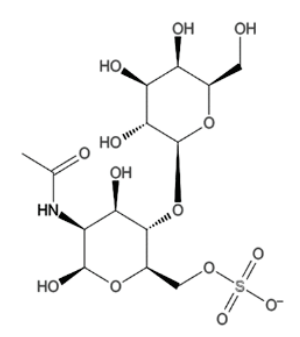

77270363

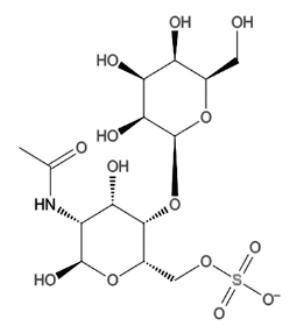

35636232<smiles>CC(=O)N[C@H]1[C@@H](O)O[C@H](CO)[C@H](O)[C@H]1O[C@H]1O[C@H](COS(=O)(=O)[O-])[C@@H](O)[C@H](O)[C@H]1O</smiles>

64634013

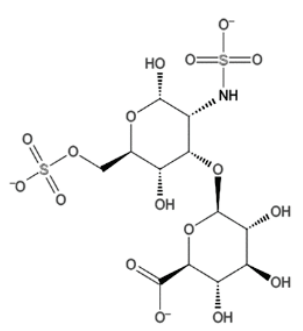

30160056

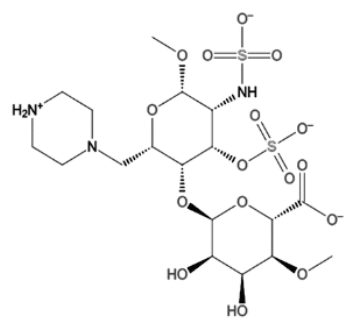

43920281

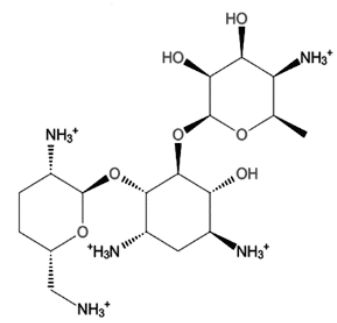

72112226<smiles>O=S(=O)([O-])N[C@@H]1[C@H](OS(=O)(=O)[O-])[C@H](O)[C@@H](CO)O[C@H]1O</smiles>

34853727<smiles>COC1OC(CO)C(CC2OC(C(=O)O)C(C)C(O)C2O)C(NC(=O)O)C1O</smiles>

56871042

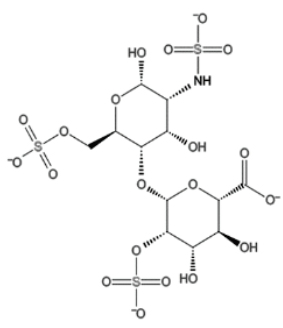

72693278

Supplement Fig. 1. The 2D representation of the small molecules identified as an agonist against sclerostin.

To further characterize the physiochemical properties (ADME/T) of the nine compounds, Qikprop simulation was performed on the identified compounds using Schrodinger software (QikProp version 3.5, Schrodinger 2012). Compounds that have high molecular weights are likely to have low solubility and have difficulty passing through the cell membrane. In our study, the molecular weight of all identified compound are within an acceptable range to have a high solubility and a high probably of being able to enter a cell. Similarly, lipophilicity (a ratio of the molecule's solubility in octanol to solubility in water (QPlogPo/w), an analysis that determines how a compound is distributed within the body after absorption and how rapidly it is metabolized and excreted, was performed on the identified compounds. This analysis revealed that all the values of compounds (C2 to C9) are within the standard range. In addition, the human oral absorption rate of each compound was determined. We found that seven of the nine compounds ( $\mathrm{C} 1$ to $\mathrm{C} 6$ and $\mathrm{C} 9$ ) were within the acceptable range generally observed for drugs while compound 34853727 and compound 64634013 fell outside the acceptable range (Table 1A). Importantly, the identified compounds demonstrated small variations within the ADME/T properties but all compounds fell within an acceptable range defined for human use. In addition to ADEM/T, we also performed density functional theory analysis on the identified compounds to determine the molecular stability of the compound which is important for achieving a biological function. The small HOMO-LUMO gap (HLG) suggests reasonable stability of all the identified compounds (Table 1A). Subsequently, we also analyzed the toxicity and druglikeness properties with OSIRIS Property Explorer software ("OSIRIS property explorer, www.organicchemistry.org/prog/peop/,"). Though the identified compounds showed acceptable ADME/T properties and drug-like scores (a combination of CloP, LogS, TPSA analysis), toxicity screening results revealed that the identified compounds possess varying risks of mutagenesis and tumorigenesis (Table 1B). In particular, three compounds $(30160056,56871042,72112226)$ that 
showed a high docking score, ADME/T properties and oral absorption rates possessed a medium to high mutagenesis rate and a high risk for tumorigenesis. While the compound 43920281 showed a moderate docking score and compounds (34853723, 64634013, 35636232) showing a low docking score, possessed a very low risk for toxicity based on parameters when compared to other identified compounds. Thus, data from these analyses suggest that further experiments should be focused on compounds that have moderate and low docking scores with a low risk of toxicity.

Table 2. Binding of small molecules with amino acids of loop2 region of sclerostin.

\begin{tabular}{|c|c|c|c|c|c|c|c|c|c|}
\hline $\begin{array}{l}\text { Amino acids } \\
\text { interactions }\end{array}$ & 30160056 & 56871042 & 72112226 & 43920281 & 77270363 & 72693278 & 34853727 & 64634013 & 35636232 \\
\hline $\mathrm{Arg} 89 \mathrm{NH}_{2} \ldots \mathrm{SO}_{3}$ & + & - & - & - & - & - & - & - & - \\
\hline Trp101 NH-PO & + & + & - & - & - & - & - & + & - \\
\hline Arg102NH-PO(2) & + & - & - & - & - & - & - & + & - \\
\hline OH-CO Lys99 & + & - & - & - & - & - & - & - & - \\
\hline Arg89 NH-OC(2) & + & - & - & - & - & - & - & - & - \\
\hline Arg89 NH-PO(2) & - & + & - & - & - & - & - & - & - \\
\hline OH-CO Ile95 & - & + & + & + & - & + & + & - & + \\
\hline Lys99 N-H...OC & - & - & + & - & - & - & - & - & - \\
\hline Arg97 N-H...OC & - & - & + & - & - & - & - & - & - \\
\hline $\operatorname{Arg} 102 \mathrm{NH}_{2}-\mathrm{PO}_{3}(2)$ & - & - & + & - & - & - & - & - & - \\
\hline Arg89 NH-PO & - & - & + & & - & - & - & - & - \\
\hline Arg97 NH-PO & - & - & - & + & + & - & - & - & - \\
\hline Arg89 $\mathrm{NH}-\mathrm{CO}_{2}(2)$ & - & - & - & + & - & - & - & - & - \\
\hline Arg102 NH-OH & - & - & - & + & - & - & - & - & - \\
\hline Gly98 NH-PO & - & - & - & - & + & - & - & - & - \\
\hline Lys99 NH-CO & - & - & - & - & + & - & - & - & - \\
\hline Arg102 NH-CO & - & - & - & - & + & - & - & - & - \\
\hline Asn93 NH-PO & - & - & - & - & + & - & - & - & - \\
\hline Asn89 NH-PO & - & - & - & - & + & - & - & - & - \\
\hline Arg89 NH-PO & - & - & - & - & - & + & - & - & - \\
\hline Arg102 NH-CO(2) & - & - & - & - & - & + & - & - & - \\
\hline Leu91 NH-CO & - & - & - & - & - & + & - & - & - \\
\hline NH-OC Arg89 & - & - & - & - & - & - & + & - & - \\
\hline NH-CO Ala89 (2) & - & - & - & - & - & - & + & - & - \\
\hline NH-CO Asn93 & - & - & - & - & - & - & + & - & - \\
\hline Asn93 NH-OH & - & - & - & - & - & - & - & + & - \\
\hline OH-CO Asn93 & - & - & - & - & - & - & - & + & - \\
\hline NH-OC Ile95 & - & - & - & - & - & - & - & + & - \\
\hline Trp101 NH-OH & - & - & - & - & - & - & - & - & + \\
\hline Arg89 NH2-CO (3) & - & - & - & - & - & - & - & - & + \\
\hline Arg102 NH-PO & - & + & - & - & - & - & - & - & - \\
\hline
\end{tabular}

+ corresponds to interaction, - reflects no interaction

Additionally, we extended our study by determining whether the identified compounds show similar or different amino acid interactions in the loop2 region of sclerostin using Pymol software (Version 1.5.0.4 Schrodinger, LLC). Our analysis revealed that the identified compounds differ in their interactions with amino acids in the loop2 region of sclerostin. We found that one compound 77270363 showed 6 interaction sites, 3 compounds (30160056, 72112226 and 64634013) had 5 interaction sites and 4 compounds (56871042, 43920281, 
72693278, 34853727) had 4 interaction sites within the loop2 region of sclerostin. In addition, we found one compound (35636232) with only 3 interactions (Table 2). Overall, these data suggest that even though these compounds differ in the interactions with amino acids in the loop2 region of sclerostin, they all have the potential to alter sclerostin function. To this end, studies have shown that the_amino acids (Pro92, Asp93, Ala94, Ile95, Gly96) in the loop2 region of sclerostin, identified as the PNAIG motif binds specifically to LRP5/6 and thereby blocks the WNT signaling pathway (Kim et al. 2013). In particular, it has been shown that this inhibitor effect is largely mediated by an interaction with two amino acids (Asn93 and Ile95) in the PNAIG region of the loop2 site of sclerostin (Holdsworth et al. 2012). In our study, the induced fit docking analysis and Pymol software found that the eight out of nine compounds interact with the same amino acids, Ile95 or Asn93, respectively, in the PNAIG sequence of the loop2 region of sclerostin that blocks WNT signaling (Table 2). This computational analysis substantiates that the identified compounds could act as potent anti-sclerostin therapeutic drugs.

\section{References}

ARDAWI MS, ROUZI AA, AL-SIBIANI SA, AL-SENANI NS, QARI MH, MOUSA SA: High serum sclerostin predicts the occurrence of osteoporotic fractures in postmenopausal women: the Center of Excellence for Osteoporosis Research Study. J Bone Miner Res 27: 2592-2602, 2012.

AUGUSTINE M, HORWITZ MJ: Parathyroid hormone and parathyroid hormone-related protein analogs as therapies for osteoporosis. Curr Osteoporos Rep 11: 400-406, 2013.

BHUTANI G, GUPTA MC: Emerging therapies for the treatment of osteoporosis. J Midlife Health 4: 147-152, 2013.

CLARKE BL: Anti-sclerostin antibodies: utility in treatment of osteoporosis. Maturitas 78: 199-204, 2014.

DAS D, KOH Y, TOJO Y, GHOSH AK, MITSUYA H: Prediction of potency of protease inhibitors using free energy simulations with polarizable quantum mechanics-based ligand charges and a hybrid water model. J Chem Inf Model 49: 2851-2862, 2009.

DE VERNEJOUL MC, KORNAK U: Heritable sclerosing bone disorders: presentation and new molecular mechanisms. Ann N Y Acad Sci 1192: 269-277, 2010.

DRAKE MT, CLARKE BL, LEWIECKI EM: The pathophysiology and treatment of osteoporosis. Clin Ther 37: 1837-1850, 2015.

GOLOB AL, LAYA MB: Osteoporosis: screening, prevention, and management. Med Clin North Am 99: 587-606, 2015.

HOLDSWORTH G, SLOCOMBE P, DOYLE C, SWEENEY B, VEVERKA V, LE RICHE K, ROBINSON MK: Characterization of the interaction of sclerostin with the low density lipoprotein receptor-related protein (LRP) family of Wnt co-receptors. J Biol Chem 287: 26464-26477, 2012.

IWAMOTO J: Vitamin K(2) therapy for postmenopausal osteoporosis. Nutrients 6: 1971-1980, 2014.

KIM JH, LIU X, WANG J, CHEN X, ZHANG H, KIM SH, HE TC: Wnt signaling in bone formation and its therapeutic potential for bone diseases. Ther Adv Musculoskelet Dis 5: 13-31, 2013.

KOBAYASHI T, KRONENBERG HM: Overview of skeletal development. Methods Mol Biol 1130: 3-12, 2014. 
LAKTASIC-ZERJAVIC N: The role of vitamin D and calcium in the management of osteoporosis. Reumatizam $\mathbf{6 1}$ : 80-88, 2014.

LEE C, YANG W, PARR RG: Development of the Colle-Salvetti correlation-energy formula into a functional of the electron density. Phys Rev B Condens Matter 37: 785-789, 1988.

LEWIECKI EM: Sclerostin: a novel target for intervention in the treatment of osteoporosis. Discov Med 12: 263-273, 2011.

LI X, OMINSKY MS, NIU QT, SUN N, DAUGHERTY B, D'AGOSTIN D, PASZTY C: Targeted deletion of the sclerostin gene in mice results in increased bone formation and bone strength. $J$ Bone Miner Res 23: 860-869, 2008.

LOMBARDI G, DI SOMMA C, VUOLO L, GUERRA E, SCARANO E, COLAO A: Role of IGF-I on PTH effects on bone. $J$ Endocrinol Invest 33: 22-26, 2010.

LYNE PD, LAMB ML, SAEH JC: Accurate prediction of the relative potencies of members of a series of kinase inhibitors using molecular docking and MM-GBSA scoring. J Med Chem 49: 4805-4808, 2006.

OLNEY RC: Mechanisms of impaired growth: effect of steroids on bone and cartilage. Horm Res 72 (Suppl 1): 30-35, 2009.

OSIRIS property explorer, www.organic-chemistry.org/prog/peop/.

PREISINGER E, ALACAMLIOGLU Y, PILS K, BOSINA E, METKA M, SCHNEIDER B, ERNST E: Exercise therapy for osteoporosis: results of a randomised controlled trial. Br J Sports Med 30: 209-212, 1996.

PRENTICE A: Diet, nutrition and the prevention of osteoporosis. Public Health Nutr 7: 227-243, 2004.

SEMINARIO J: Recent Developments and Applications of Modern Density Functional Theory 1st ed, Vol. 1. Elsevier Science, Amsterdam, 1996.

SCHRODINGER. Software, LLC, New York, http://www.schrodinger.com/citations/

SINGH KD, KIRUBAKARAN P, NAGARAJAN S, SAKKIAH S, MUTHUSAMY K, VELMURGAN D, JEYAKANTHAN J: Homology modeling, molecular dynamics, e-pharmacophore mapping and docking study of Chikungunya virus nsP2 protease. J Mol Model 18: 39-51, 2012.

VAN BEZOOIJEN RL, SVENSSON JP, EEFTING D, VISSER A, VAN DER HORST G, KARPERIEN M, LOWIK CW: Wnt but not BMP signaling is involved in the inhibitory action of sclerostin on BMP-stimulated bone formation. J Bone Miner Res 22: 19-28, 2007.

VEVERKA V, HENRY AJ, SLOCOMBE PM, VENTOM A, MULLOY B, MUSKETT FW, CARR MD: Characterization of the structural features and interactions of sclerostin: molecular insight into a key regulator of Wnt-mediated bone formation. J Biol Chem 284: 10890-10900, 2009.

WINKLER DG, SUTHERLAND MK, GEOGHEGAN JC, YU C, HAYES T, SKONIER JE, LATHAM JA: Osteocyte control of bone formation via sclerostin, a novel BMP antagonist. EMBO J 22: 6267-6276, 2003. 